\title{
Experimental and numerical investigation of turbulent flow induced pipe vibration in fully developed flow
}

\author{
Matthew T. Pittard \\ matt@pittard.org \\ R. Daniel Maynes \\ Jonathan D. Blotter \\ Robert P. Evans
}

Follow this and additional works at: https://scholarsarchive.byu.edu/facpub

Part of the Mechanical Engineering Commons

\section{Original Publication Citation}

Pittard, MatthewÂ T., RobertÂ P. Evans, DanielÂ R. Maynes, and JonathanÂA D. Blotter.

"Experimental and numerical investigation of turbulent flow induced pipe vibration in fully developed flow." Review of Scientific Instruments 75 (24): 2393-241.

\section{BYU ScholarsArchive Citation}

Pittard, Matthew T.; Maynes, R. Daniel; Blotter, Jonathan D.; and Evans, Robert P., "Experimental and numerical investigation of turbulent flow induced pipe vibration in fully developed flow" (2004). Faculty Publications. 435.

https://scholarsarchive.byu.edu/facpub/435 


\title{
Experimental and numerical investigation of turbulent flow induced pipe vibration in fully developed flow
}

\author{
Matthew T. Pittard \\ Department of Mechanical Engineering, Brigham Young University, Provo, Utah 84602 \\ Robert P. Evans \\ Idaho National Engineering and Environmental Laboratory, Idaho Falls, Idaho 83415-3790 \\ R. Daniel Maynes and Jonathan D. Blotter ${ }^{a)}$ \\ Department of Mechanical Engineering, Brigham Young University, Provo, Utah 84602
}

(Received 11 November 2003; accepted 4 April 2004; published online 23 June 2004)

\begin{abstract}
Flow-induced pipe vibration caused by fully developed pipe flow has been observed but not fully investigated when turbulent flow prevails. This article presents experimental results that indicate a strong correlation between the volume flow rate and a measure of the pipe vibration. In this work, the standard deviation of the frequency-averaged time-series signal, measured using an accelerometer attached to the pipe, is used as the measure of pipe vibration. A numerical, fluid-structure interaction (FSI) model used to investigate the relationship between pipe wall vibration and the physical characteristics of turbulent flow is also presented. This numerical FSI approach, unlike commercial FSI software packages, which are based on Reynolds averaged Navier-Stokes flow models, is based on large eddy simulation (LES) flow models that compute the instantaneous pressure fluctuations in turbulent flow. The results from the numerical LES models also indicate a strong correlation between pipe vibration and flow rate. In general, the numerical simulations show that the standard deviation of the pipe wall vibration is proportional to the pressure fluctuations at the wall induced by the flow turbulence. This research, indicates that the pressure fluctuations on the pipe wall have a near quadratic relationship with the flow rate. Furthermore, the experimental results and the numerical modeling show that there is a definite relationship between the acceleration of the pipe (pipe vibration) and the flow rate. These last two concepts open possible avenues for the development of a non-intrusive flow sensor. (C) 2004 American Institute of Physics. [DOI: $10.1063 / 1.1763256]$
\end{abstract}

\section{INTRODUCTION}

Flow measurements are used in many applications and for various purposes. Some of these include providing data for system control, process analysis, accounting of yield, and consumption. Recent developments in technology have improved sensor designs and measurement techniques. Coriolis, magnetic, and ultrasonic flow meters are a few examples of this improved technology. Although many high-quality, pipe-flow sensors and measurement techniques exist, there is a need for the development of a low-cost, nonintrusive, flow sensor. The geothermal industry is in particular need of such a sensor for the measurement of brine flows. Geothermal brine typically consists of hot, pressurized liquid, carrying dissolved solids. As the pressure of the liquid drops, some of the dissolved solids precipitate out of solution coating any surface in contact with the fluid. This coating renders an intrusive flow meter inoperable after a short time. Similar non-intrusive measurements are also needed in the nuclear industry.

A promising solution to the development of a nonintrusive flow meter is through the use of a sensor based on the signal fluctuations (i.e., the standard deviation) from a vibra-

${ }^{\text {a)} E l e c t r o n i c ~ m a i l: ~ j b l o t t e r @ b y u . e d u ~}$ tion sensor such as an accelerometer or microphone attached to the outside surface of the pipe. The method was first considered at the Idaho National Engineering and Environmental Laboratory (INEEL) as a diagnostic tool for pump performance in nuclear applications. ${ }^{1}$ During the Loss-of-Fluid Test nuclear reactor safety testing program, measurements were analyzed to determine their uncertainty and limitations. It was noticed that in turbulent single-phase flow, the standard deviation of the signal from a pipe mounted accelerometer increased with flow rate. ${ }^{2}$ Based on these results, initial laboratory tests were performed by the authors. These tests demonstrated that it was possible to use signal noise from an accelerometer to sense flow noise in a pipe. ${ }^{3,4}$ Two basic methods were initially investigated. The first consisted of looking at shifts in the frequency domain due to changes in the flow rate. These results compared with theory but the frequency shifts are very small. For example, for a flow rate change from 7000 to $22000 \mathrm{gm} / \mathrm{s}$, a frequency shift of approximately $0.2 \mathrm{~Hz}$ is detected. The second method consisted of investigating shifts in the standard deviation of the time series signal and is the focus of this article. These preliminary results were used to obtain funding from the Department of Energy through an INEEL Laboratory Directed Research and Development award to construct an air-water flow 
loop, and to continue characterizing the relationship between accelerometer signal standard deviation and flow rate.

In turbulent flow, the relative motion of the fluid in the boundary layer generates flow disturbances in the form of vortices or eddies. As the flow rate increases so does the amount of turbulence. There is a continuous transfer of energy from the main flow into large eddies, and from the large eddies into smaller eddies, which dissipate most of the energy. This process occurs in a narrow strip inside the boundary layer, in the neighborhood of the wall. This energy dissipation produces large kinetic energy losses in the fluid. As the fluid molecules in the vortices go from locations of higher kinetic energy to regions of lower kinetic energy, (i.e., from near the edge of the boundary layer to near the wall), the kinetic energy of the fluid is converted into heat and potential energy in the form of pressure. These pressure fluctuations excite vibratory oscillations in the pipe through which the fluid is flowing. The movement of the pipe also causes additional pressure fluctuations in return. This twoway interaction results in flow-induced vibration. The measurement concept presented in this article is based on the flow-induced vibration measurements obtained from an accelerometer mounted to the surface of the pipe.

The vibration of a pipe transporting fluid has been recognized by researchers and quantified using numerical, analytical, and experimental techniques. In the past, researchers such as Saito, Miyano, and Furukawa, ${ }^{5}$ Durant et al. ${ }^{6}$ Durant and Robert, ${ }^{7}$ Brevart and Fuller, ${ }^{8}$ and Kim and $\mathrm{Kim}^{9}$ have presented relationships between flow rate and pipe vibration. Although results vary, each researcher proposed that pipe vibration was a direct result of the pressure fluctuations at the pipe wall inherent in turbulent flow.

Although some results have been presented as listed above, researchers still face basic challenges in attempting to solve this problem. Experimental solutions can provide an accurate representation of the physical phenomena but they are inherently time consuming and expensive and a detailed experimental investigation of the vibrational pipe response to fully developed turbulent flow has not yet been presented. This will be one focus of the present study. Current numerical and analytical solutions typically model the fluid flow using simplifying assumptions, which do not provide instantaneous values for fully developed pipe flow. For example, commercial fluid structure interaction codes use Reynolds Averaged Navier-Stokes (RANS) equations to model the turbulent flow. These models are based on time averages and do not compute the pressure variations at the fluid-structure interface. Since it has been determined that these pressure variations contribute to the pipe vibrations, commercial RANS based codes cannot accomplish the purpose of this study. Instead of the RANS based models, large eddy simulation (LES), which more closely models the actual physics of the flow is used. The LES approach is derived from direct numerical simulation (DNS) which effectively integrates the Navier-Stokes equations. The physics and the assumptions behind LES modeling are discussed in the article.

The purpose of this research is to experimentally and numerically quantify the relationship between flow rate and pipe vibration and to determine if the relationship is strong

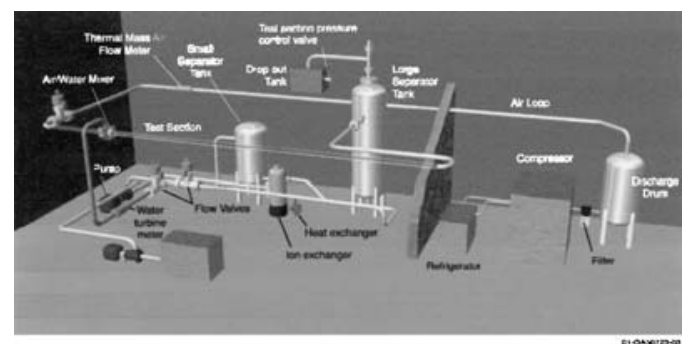

FIG. 1. Layout of the experimental flow loop used for all experiments.

enough to pursue the development of an accelerometer based, nonintrusive flow sensor.

\section{EXPERIMENT}

This section first describes the experimental test facility and the data acquisition process. The experimental results are then presented and discussed.

\section{A. Experimental test facility}

An air-water flow loop was developed for this research. Although the research is focused on single phase (liquid) flow, the flow loop was designed to have two phase (airwater) flow capability. A diagram and a schematic of the flow loop are shown in Figs. 1 and 2, respectively. This flow loop consists of five main components which are briefly described.

\section{Water system}

The water system supplies the liquid phase of the test fluid and consists of a primary pump, air- and manuallyoperated flow control valves, a test loop, a bypass loop with a water clean-up system, two air-water separator tanks with an air discharge system, plus the interconnecting piping, loop pressure, and pump speed instrumentation.

Water is circulated through the flow loop by a Goulds 10-in. diameter impeller, centrifugal pump driven by a Reliance 75 HP, $2500 \mathrm{rpm}$, Model Super RPM dc, direct current motor with a variable-speed controller. The flow path for the water is controlled by two air-operated Valtek, size 3, Class

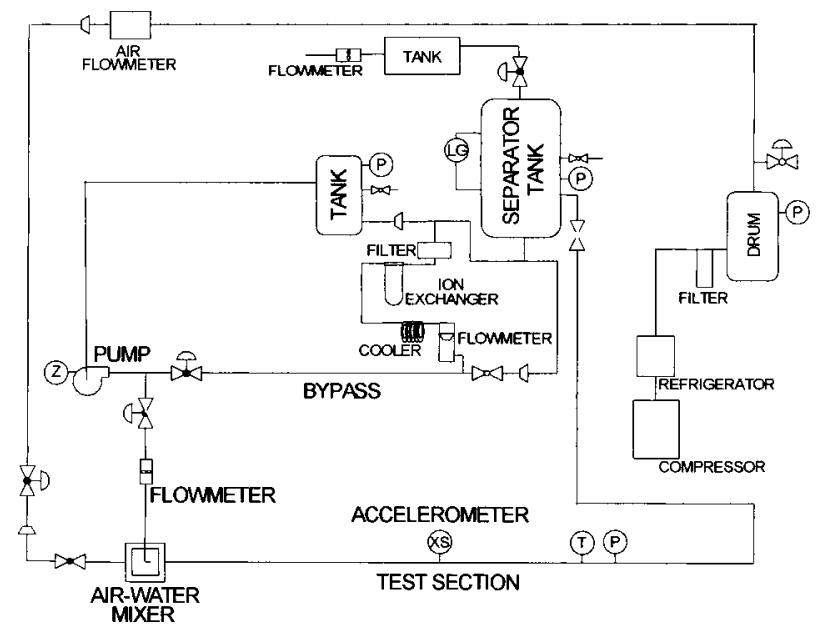

FIG. 2. Schematic of the flow loop utilized for all experiments. 
150 valves at the outlet of the pump. Each valve opening can be controlled to allow the desired flow through either the test section or the bypass line.

The water for the test section goes from the pump, through the control valve, and then through an air-water mixer, where it could be mixed with air from the air supply system before entering the test section. After passing through the test section, the water enters the first of two air-water separator tanks, which allow any entrained air to separate from the water by gravity. In the first tank, the air can be exhausted to the atmosphere through a pressure control valve and drop-out tank. A turbine flow meter measures the flow rate of the exhausted air. The separator tank exhaust control valves control the amount of air exhausted, thus controlling the pressure in the first tank as well as the pressure in the loop and test section. A pressure gauge and level indicator, respectively, monitor the pressure and water level in the tank. The water from the first air-water separator tank passes out of the bottom of the tank into a second, smaller air-water separator tank, where any additional air can be removed from the water. The water then goes to the pump to start the cycle again.

The bypass loop enables two functions: It provides an alternate path for the water so that the amount of water flowing through the test section can be controlled without changing the pump speed, and it also provides a system to cool and clean the loop water. The cooling is accomplished by passing the water through a heat exchanger, which transfers some of the heat to secondary cooling water. The water also passes through an ion exchanger and filter to clean the water, before reentering the flow path between the first and second airwater separator tanks. The amount of water passing through the cooling and cleanup system is monitored by a variablearea rotameter and controlled by a hand-operated valve in the bypass line. The remainder of the water from the bypass loop reenters the flow path at the first air-water separator tank. The bypass loop piping is primarily 0.0762 or $0.102 \mathrm{~m}$ ( 3 or 4 in.) nominal pipe size and primarily of drawn 304 stainless steel and polyvinyl chloride (PVC).

Normal system operation consists of turning on the air compressor and pressurizing the air supply system to a given level. The loop is then filled with deionized water. The system is slowly started from an uncirculated, cold-water condition. The water is allowed to run until the system piping is free of air, an equilibrium temperature has been reached, and the flow rate is steady.

\section{Control system}

The pump and valves in the flow loop are controlled from a control panel in an adjacent room. The control panel has an on-off switch for the pump motor as well as a variable speed control. A dial gauge, which receives input from the pump speed controller, displays the speed in revolutions per minute. The air-operated valves are controlled by electric potentiometers on the control panel. The panel is situated so that the test loop can be viewed during operation.

\section{Test measurement transducers}

The flow loop sensors consisted of the following:
- PCB Piezotronics Model 352B68 piezoelectric accelerometer (resolution $0.003 \mathrm{~m} / \mathrm{s}^{2}$ with less than $1 \%$ error) to measure pipe vibration in the test section.

- Flow Technology FT Series full-bore turbine flow meter with a $0.0762 \mathrm{~m}$ (3 in.) nominal diameter, operating range of 250 to 2500 liters $/ \mathrm{min}(1 / \mathrm{m})$, repeatability of $\pm 0.5 \%$ of reading, and linearity of $\pm 0.5 \%$ of reading to measure water flow through the test section.

- 0 to $1.034 \mathrm{MPa}$ (0 to $150 \mathrm{psig}$ ) Heise pressure gauge to measure test section pressure.

- Bimetallic thermometer temperature gage to measure the test section temperature.

\section{Test section}

The flow loop test section consisted of a $6.1 \mathrm{~m}$ (240 in.) interchangeable section of pipe supported at $1.1 \mathrm{~m}$ intervals. In this effort, five different test sections were used. All piping utilized in the test section was hydraulically "smooth." The first three sections, were nominal $0.0762 \mathrm{~m}$ (3 in.) diameter schedule 40 pipe [actual diameter is $D=0.0779 \mathrm{~m}$ (3.068 in.) with a wall thickness of $t=5.48 \times 10^{-3} \mathrm{~m}(0.216$ in. $\left.)\right]$ made of clear PVC, drawn 304/304L stainless steel, and drawn aluminum. These three sections were utilized to determine the effects of material properties on the accelerometer standard deviation-flow rate relationship. Two other test sections were also employed. The first was $0.1016 \mathrm{~m}$ (4 in.) nominal diameter schedule 40 clear PVC pipe $[D$ $=0.1023 \mathrm{~m}$ (4.026 in.) and $t=6.02 \times 10^{-3} \mathrm{~m}(0.237 \mathrm{in}$.) $]$. The other section was $0.0381 \mathrm{~m}$ (1.5 in.) diameter drawn stainless steel schedule 40 pipe $[D=0.041 \mathrm{~m}$ (1.61 in.) and $t=3.68 \times 10^{-3} \mathrm{~m}(0.145 \mathrm{in}$.) $]$. These sections were employed to investigate and characterize the effects of pipe diameter. The various test sections were inserted into the flow loop independently, utilizing flanged connections. For all cases the accelerometer was mounted on top of the pipe test section $2.34 \mathrm{~m}$ (92 in.) downstream of the inlet. The longest hydrodynamic development length existed for the 0.1016 diameter pipe and was estimated to be approximately $0.8 \mathrm{~m}$. The pipe was fixed in all three directions at the two ends and was supported with a suspension rod at four equally spaced intermediate distances. The ends of the test section were mounted in rubber isolators to help provide vibration isolation to the test section. It is important to note that different boundary conditions would effect the response of the pipe. Therefore, each pipe arrangement would need some form of calibration.

\section{B. Data acquisition}

Data from the flow loop transducers were recorded simultaneously on a HP35670A Spectrum Analyzer and a PC based data acquisition system. The accelerometer data were recorded using the spectrum analyzer while all other data were recorded using the PC data acquisition system.

The accelerometer data were initially acquired using various sample rates and time spans. Based on these results, a $2 \mathrm{~s}$ time span and a sample rate of 2048 samples/s was used 


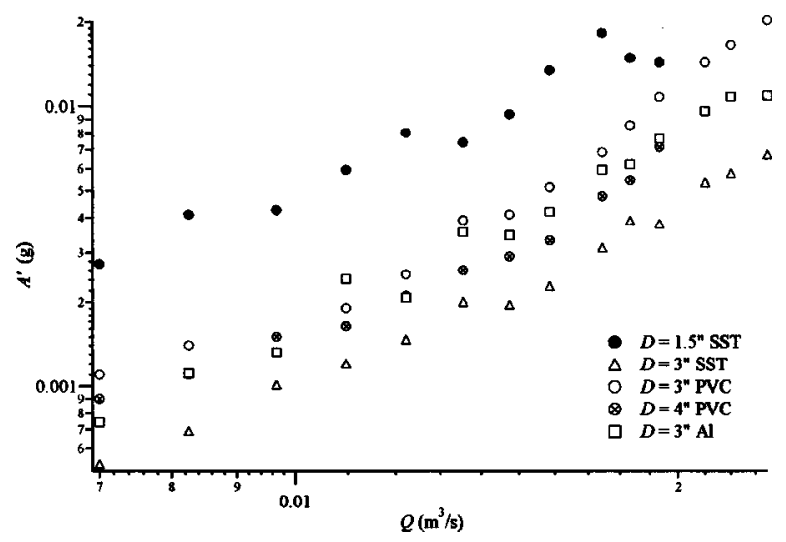

FIG. 3. $A^{\prime}$ plotted as a function of volume flow rate for five pipe sections with diameters of $1.5,3$, and 4 in. and stainless steel, PVC, and aluminum pipes.

to acquire the data presented. Antialiasing filters were used with a cut off frequency of $800 \mathrm{~Hz}$. The accelerometer time series data were transformed to the frequency domain using a fast Fourier transform. Ten data sets were averaged in the frequency domain and the data was transformed back to the time domain. Initially, 50 data sets were used to compute the average. However, the difference between the results based on 50 data sets were indiscernible at all flow rates. Therefore, only 10 data sets were used in the averaging. The standard deviation of the frequency domain average time-series signal was then used to compare with the flow rate.

Fourteen volume flow rate, $Q$ between 0.4 and 1.5 $\mathrm{m}^{3} / \mathrm{min}(400-1500$ liters $/ \mathrm{min})$ were used for each of the three $0.0762 \mathrm{~m}$ ( $3 \mathrm{in}$.) test sections. The minimum flow rate of $0.4 \mathrm{~m}^{3} / \mathrm{min}$ was required to keep the pipe full of water. The $1.5 \mathrm{~m}^{3} / \mathrm{min}$ limit was the highest flow rate obtainable with the pump and system components. The flow steps were repeatable within a $\pm 0.002 \mathrm{~m}^{3} / \mathrm{min}$ range and the flow rate was held relatively constant with a maximum fluctuation of $\pm 0.001 \mathrm{~m}^{3} / \mathrm{min}$ during testing (less than $1 \%$ error). The same accelerometer position, data acquisition rate, and volume flow rates were used for each of the test sections.

\section{Results and analysis}

One focus of this research was to determine the relationship between volume flow rate and the rms of the pipe vibration signal measured with an accelerometer and to determine if, based on this relationship, a nonintrusive flow rate sensor or measurement technique could be developed. This section presents the results of the experiments performed to characterize this relationship. Shown in Fig. 3 on a log scale is the rms of the accelerometer signal, or the typical fluctuations in the acceleration due to the pipe vibration $A^{\prime}$ plotted versus volumetric flow rate for all five test sections considered. $A^{\prime}$ is dimensionless and is measured in multiples of the acceleration of gravity $g$. The standard deviation computed from a set of ten independent measurements was less than $3.0 \%$ of the measured value for each data point shown in Fig. 3.

Several trends of interest are evident from the data of Fig. 3. First, as expected $A^{\prime}$ increases with increasing $Q$ for

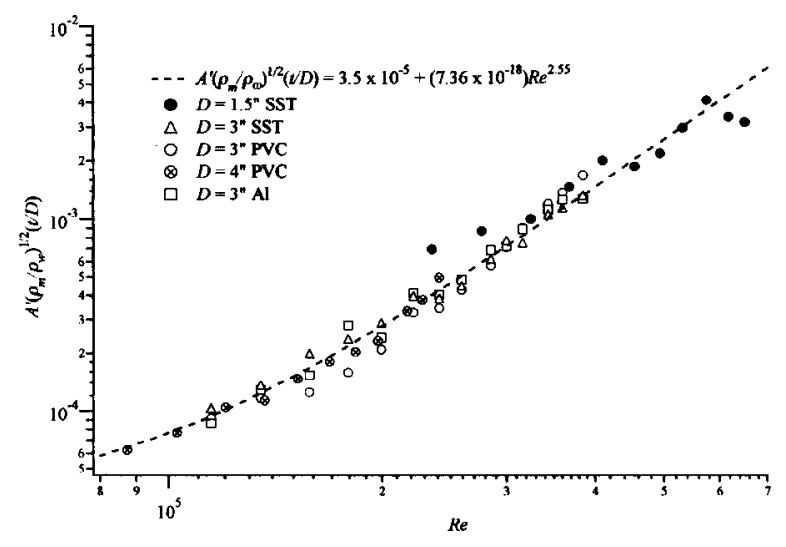

FIG. 4. $A^{\prime}\left(\rho_{m} / \rho_{w}\right)^{1 / 2}(t / D)$ shown as a function of the Reynolds number for all five pipes considered.

all materials and all pipe diameters. The increase appears to be power law in form. Second, at a given flow rate, and for the same pipe material, $A^{\prime}$ is observed to increase as the pipe diameter $D$ decreases. This is evident in data for the 1.5 and 3 in. stainless steel pipes where for a given flow rate $A^{\prime}$ is nominally four times greater for the 1.5 in. pipe. It is also manifest in the 3 and 4 in. PVC pipes. Third, the data indicate that for a given pipe diameter the magnitude of $A^{\prime}$ decreases with increasing pipe material density. For the 3 in. diameter pipes $A^{\prime}$ is smallest for the stainless steel pipe and is greatest for the PVC pipe with the data for the aluminum pipe residing in between. The material densities are $\rho_{m}$ $=7800,2700$, and $1400 \mathrm{~kg} / \mathrm{m}^{3}$ for the stainless steel, aluminum, and PVC pipes respectively. The data of Fig. 3 suggest that $A^{\prime}$ is proportional to $\left(1 / \rho_{m}\right)^{1 / 2}$.

Shown in Fig. 4 is the quantity $A^{\prime}\left(\rho_{m} / \rho_{w}\right)^{1 / 2}(t / D)$ plotted on a log scale as a function of the Reynolds number Re $=\rho_{w} \overline{V D} / \mu . \rho_{w}$ and $\mu$ are the flowing water density and viscosity respectively, $t$ is the pipe wall thickness, and $V$ is the average fluid velocity, equal to $Q / A_{c}$ where $A_{c}$ is the pipe cross-sectional area. When normalized in this fashion the data, for all pipe diameters and materials investigated here, collapse and are well represented by the power law curve fit shown in the figure. This expression is also given below as Eq. (1):

$A^{\prime}\left(\rho_{m} / \rho_{w}\right)^{1 / 2}(t / D)=3.5 \times 10^{-5}+\left(7.36 \times 10^{-18}\right) \operatorname{Re}^{2.55}$.

This relation shows that estimation of the volumetric flow rate through a pipe based on direct measurement of the fluctuations in the pipe vibration signal is possible. Of course, Eq. (1) is based only on the three pipe materials and three pipe diameters explored here, in addition to the particular pipe mounting approach. Additional experiments will be necessary to expand the results over a broader range of problem variables. Based on these results, however, the development of a nonintrusive flow meter based on this methodology seems quite promising.

\section{NUMERIC}

As noted previously in Sec. I several investigators have concluded that pipe vibration is a direct result of the pressure fluctuations at the pipe wall inherent in turbulent flow. Nu- 
merically modeling the turbulent flow field and importing the results of the induced pressure fluctuations into a structural model would provide a simulation of an experimental setup and would allow direct comparison between the flow physics and the structural response. This is the focus of the following section.

\section{A. Turbulent pipe flow simulation}

The turbulent flow of an incompressible Newtonian fluid with constant viscosity is completely described mathematically by the Navier-Stokes equations coupled with the continuity equation. Numerical approaches to model turbulent flows are also based on solutions of these equations. The direct numerical simulation (DNS) approach attempts to discretize the flow into sufficiently small volumes so that all turbulent length and time scales of the flow are resolved. At large Re, however, the necessary discretized volumes are so small and the total number of cells so large that it makes large $\mathrm{Re}$ flows unrealistic to solve in this manner at the present. $^{10}$ The classical approach to analyzing turbulent flows follows the "time averaging" method proposed by Reynolds and is often called the Reynolds Averaged NavierStokes (RANS) approach. In this method the velocity and pressure terms in the Navier-Stokes and continuity equations are separated into time-averaged mean and fluctuating components and then the equations are themselves averaged over time. The resulting time-averaged equations resemble the Navier-Stokes equations except for the addition of a cross-correlation term, known as the Reynolds stress. The Reynolds stress is a product of two fluctuating components of the flow field and is an unknown, thus the problem is no longer "closed." A variety of empirical modeling techniques have been developed to address this closure problem. However, these approaches can only yield time averaged quantities. This approach is thus not adequate for determining the instantaneous pressure distributions on the wall in fully developed turbulent pipe flow. Since the present interest is to obtain the instantaneous properties of the flow field and import the pressure loading into a finite element solver, RANS based techniques will not provide the needed results.

In contrast to a time-averaged approach, large eddy simulation (LES) provides a model which computes the instantaneous velocity and pressure fields. The LES approach is similar to DNS except the equations are "spatially filtered" to the size of the grid. This means that the flow is resolved to a characteristic scale, usually taken to be the size of the grid, and then modeled on the smaller scales. The motivation for this comes from the fact that large eddies possess an anisotropic behavior and need to be resolved while at the smallest scales the turbulence is locally isotropic and can be treated adequately from a statistical standpoint. Length scales the size of the grid or larger are known as the grid scale (GS) and scales smaller than that are referred to as subgrid scales (SGS). Typically, the grid spacing is such that the majority of the total turbulent kinetic energy contained in the large eddies is directly computed. The remaining fraction of the kinetic energy that is not resolved to the GS must be modeled. $^{11}$
Instead of time-averaged quantities, spatially or locally averaged values $\tilde{u}_{i}$ are obtained. The governing equations for LES flow are in Eqs. (2) and (3):

$$
\begin{aligned}
& \frac{\partial \tilde{u}_{i}}{\partial x_{i}}=0 \\
& \frac{\partial \tilde{u}_{i}}{\partial t}+\frac{\partial}{\partial x_{j}}\left(\tilde{u}_{i} \tilde{u}_{j}\right)=-\frac{1}{\rho} \frac{\partial}{\partial x_{i}} \tilde{P}+\nu \frac{\partial^{2} \tilde{u}_{i}}{\partial x_{j}^{2}}+\frac{\partial \tau_{i j}}{\partial x_{j}} .
\end{aligned}
$$

$\tau_{i j}$ is the stress tensor which represents the SGS contributions to the overall GS velocity. It is a term similar to the Reynolds stress term obtained using the RANS approach and it is defined as the difference of the local average of the product of the instantaneous velocities and the product of the local averages as shown in Eq. (4):

$$
\tau_{i j}=\overline{u_{i} u_{j}}-\tilde{u}_{i} \tilde{u}_{j} \text {. }
$$

$\tau_{i j}$ is modeled on the SGS and the accuracy of the model falls on the assumption that turbulence smaller than the size of the grid is homogeneous and accurately modeled. Since the accuracy of LES is largely a function of the resolution of the large eddies, as the Reynolds number increases so does the spectrum of eddies which requires increasingly finer meshes to resolve the majority of the turbulent kinetic energy. Therefore, with LES there is a trade off between computation cost and model accuracy. However, if various constraints are followed an acceptable balance can be obtained.

As with the Reynolds stress terms in the RANS approach, the SGS stress $\tau_{i j}$ must be modeled since there are no transport equations to allow computation of the local average of the velocity products. It is mathematically modeled by relating the subgrid stress with a turbulent viscosity and strain rate as shown in Eq. (5):

$$
\tau_{i j}-\frac{1}{3} \delta_{i j} \tau_{k k}=-2 \mu_{t} S_{i j} .
$$

$\delta$ is the Kronecker delta, $S_{i j}$ represents the rate of strain tensor, and $\mu_{t}$ is the SGS eddy viscosity. The most common SGS eddy viscosity model is the Smagorinsky-Lilly model. ${ }^{12}$ In this model, the eddy viscosity is proportional to a subgrid mixing length $(\lambda)$ and the strain rate as defined by Eq. (6):

$$
\mu_{t}=\rho \lambda^{2} \sqrt{2 S_{i j} S_{i j}}
$$

Overall, Smagorinsky's model is adequate for isotropic flows but usually breaks down near boundaries unless near wall treatment is employed since the contribution of turbulent viscosity at the wall is nearly zero. Therefore, accurately accounting for the wall boundary condition requires modifications to the mixing length. The method used in this research takes the minimum of the products $\kappa d$ and $C_{s} V^{1 / 3}$ where $\kappa$ is the von Karman constant $(\kappa=0.42), d$ is the distance to the closest wall, $C_{s}$ is the Smagorinsky constant, and $V$ is the volume of the computational cell. In general, $C_{S}$ $=0.1$ yields the best results for a wide range of flows ${ }^{13}$ and was used here.

To illustrate the differences between RANS and LES, the flow field for turbulent flow in a pipe was modeled using both approaches. Figure 5 compares the fluctuations obtained 


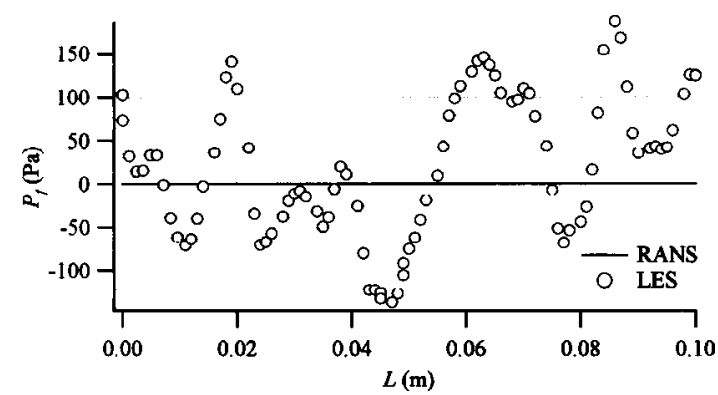

FIG. 5. Comparison of a typical wall pressure distribution along the length of a pipe, minus the mean pressure, using the RANS and LES approaches for $\operatorname{Re}=4 \times 10^{6}$ and at a constant tangential location.

from the mean pressure distributions along the length of the pipe $P_{f}$. The data correspond to a fixed tangential position on the pipe wall. Figure 5 clearly illustrates the difference between the time averaged values computed in RANS based models and the instantaneous and fluctuating values computed utilizing LES based models.

\section{B. Solution procedure}

The procedure for determining the relationship between flow rate and pipe vibration consisted of first solving the fluid mechanical problem. So that this process could be repeated by others, commercial software was used. The LES model of the flow field was obtained through the use of the commercial software package FLUENT ${ }^{\circledR}$. Once the solution to the flow field was obtained, the pressure field on the pipe wall was exported to a commercially available structural finite element package known as ANSYS ${ }^{\circledR}$. ANSYS uses the pressure field solved for in FLUENT to calculate the pipe response. This is a computationally intensive process requiring export from FLUENT and mapping in the pipe model built in ANSYS of tens of thousands of pressure data points for each time step.

Initially it was assumed that the deflections in the pipe would change the flow field and that an update of the flow model geometry would be required between time steps in the flow field solution. To verify this assumption, the displacements caused by the pressure fluctuations at the pipe wall were computed in the structural model. The displacement due purely to the turbulent flow calculated using ANSYS was shown to be on the order of nanometers $\left(1 \times 10^{-9} \mathrm{~m}\right)$. Since for all conditions considered, the viscous sublayer is much larger than these displacements it was assumed that a structural update of the deformed pipe geometry was not needed between time steps. Therefore, the deformed geometry was not included in the flow solution and the solutions were not coupled. This result could change for various pipe configurations and flow rates and should be checked in each case.

\section{Modeling results}

A 32 GB RAM, 64 processor, $400 \mathrm{MHz}$ super computer was used to solve the numerical models. The LES turbulent flow model consisted of a pipe of internal diameter $D$ $=7.62 \mathrm{~cm}$, and total length $L=10 \mathrm{~cm}$. The total number of elements in the grid domain was approximately $3 \times 10^{5}$. The

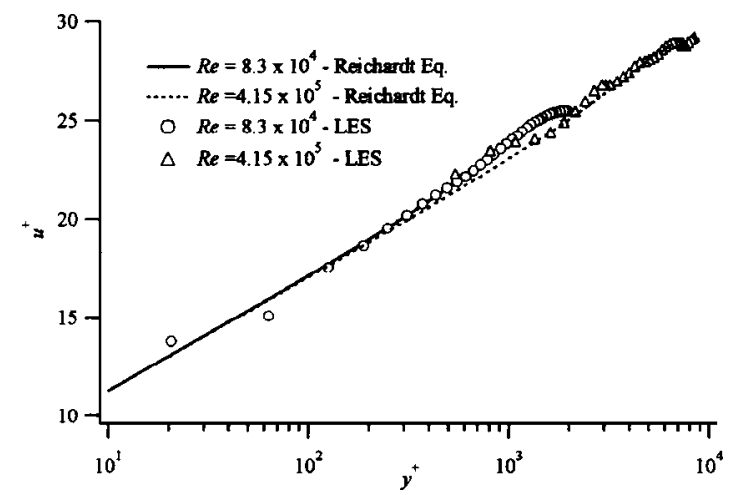

FIG. 6. Comparison of the Reichardt equation and the time averaged streamwise velocity distribution obtained from the LES model for $\mathrm{Re}=8.3$ $\times 10^{4}$ and $4.15 \times 10^{5}$.

no-slip condition was employed at the walls of the pipe and periodic boundary conditions in the streamwise direction were employed. According to Eggles, ${ }^{10}$ the streamwise pipe domain should be nominally five diameters with a resolution of $y^{+}<1$ near the wall, where $y^{+}$is the inner wall coordinate, $y^{+}=y \sqrt{\tau_{w} / \rho} / \nu$. The normal distance from the wall is $y, \rho$ is the fluid density, $\tau_{w}$ is the wall shear stress, and $\nu$ is the kinematic viscosity. Since the ranges of Reynolds numbers based on pipe diameter for this investigation were in the range $8.3 \times 10^{4}-4.15 \times 10^{5}$, an extremely fine grid would be necessary to yield resolution to within $y^{+}<1$. Grid independence studies performed by the authors showed, however, that using a minimum $y^{+}$value corresponding to the extent of the log-law layer in the time-averaged velocity distribution produced adequate results and did not significantly change the fluctuating pressure fields. The nondimensional wave number of 20 was used and $97 \%$ of the total turbulent energy was resolved by the model. This in turn specified the time step to be approximately one hundredth of the pipe length divided by the average velocity. Approximately $100 \mathrm{~h}$ of computer time was required for each simulation. Slight correlation errors were noted in the pressure and velocity fields when a pipe length of $4 / 3 D$ was used as opposed to $5 D$, however, the differences were not significant enough (less than 5\%) to change the ultimate result. The $y^{+}$and pipe domain values used are modest compared to suggested values but provide a feasible computation time and a reasonable flow model as determined by the numbers listed above.

The results of the LES model were validated by comparing the time-averaged velocity profile of the LES model with the well-established law-of-the-wall velocity distribution. Specifically the results were compared in terms of the inner coordinates $u^{+}$and $y^{+}$, with the Reichardt equation and the results are shown in Fig. $6 .{ }^{14} u^{+}=u / u_{\tau}$, where $u_{\tau}$ is the friction velocity, $u_{\tau}=\sqrt{\tau_{w} / \rho}$, and $y^{+}$is defined above. Results for the upper and lower Re explored are shown in the figure and the comparison between the Reichardt equation and the LES model are very good.

The LES model was also validated by comparing the mean pressure gradients with that obtained using a Darcy friction factor $f$, and the relation given by Eq. (7): 
TABLE I. Pressure gradient comparison.

\begin{tabular}{cccc}
\hline \hline $\begin{array}{c}\text { Flow rate } \\
\text { (liters/min) }\end{array}$ & $\begin{array}{r}\text { Theory } \\
(\mathrm{Pa} / \mathrm{m})\end{array}$ & $\begin{array}{c}\text { FLUENT } \\
(\mathrm{Pa} / \mathrm{m})\end{array}$ & \% error \\
\hline 300 & -147 & -136 & -7.48 \\
500 & -368 & -350 & -4.89 \\
750 & -764 & -694 & -9.16 \\
1000 & -1284 & -1195 & -6.93 \\
1250 & -1924 & -1764 & -8.32 \\
1500 & -2679 & -2460 & -8.17 \\
& & Average & -7.49 \\
\hline \hline
\end{tabular}

$$
\frac{\Delta P}{L}=f \frac{\rho \overline{V^{2}}}{2 D} .
$$

$f$ was obtained from the Colebrook equation given by Eq. $(8):{ }^{15}$

$$
\frac{1}{\sqrt{f}}=-0.869 \ln \left(\frac{\varepsilon}{3.7 D}+\frac{2.523}{\operatorname{Re} \sqrt{f}}\right) .
$$

$\varepsilon$ is the wall roughness and was set to zero here. The results of the comparison are summarized in Table I. For all cases considered the differences are within $9 \%$.

At each time step in the flow solution, the pressure fluctuations were exported for use in the structural model. The positive and negative pressure fluctuations on the surface of the pipe for one time step are illustrated in Fig. 7. This provides insight to the spatial distributions of the pressure loading along the pipe wall. The vertical axis is the angle measurement of the pipe (i.e., the circumferential length) and the horizontal axis represents the length of the pipe.

Although the pressure fields shown in Fig. 7 may appear random it has been well documented that turbulence is not a random phenomena. ${ }^{11-13}$ Analysis of the pressure fluctuation data at the pipe wall, shows that a near normal distribution exists. This is shown in Fig. 8. Since the main goal of the (CFD) simulation is to obtain the pressure fluctuations, avenues to obtain these pressures need not be limited to the CFD approach used here. If the pressure fluctuations always behave Gaussian the possibility exists of statistically charactering the pressure fields. Such a method could then circumvent the expensive CFD approach.

As the flow rate increases, the standard deviation of the pressure field also increases. The standard deviation of the

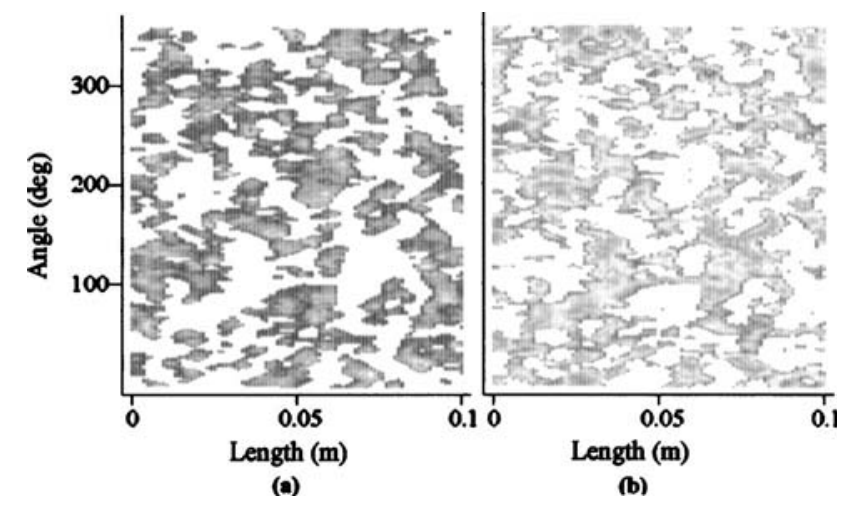

FIG. 7. Typical pressure field fluctuations on the pipe surface for (a) a positive pressure field, and (b) a negative pressure field.

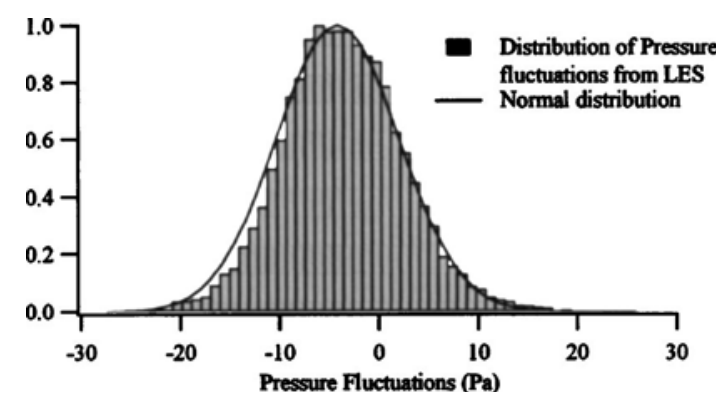

FIG. 8. Distribution of the turbulent pressure fluctuations at each interior point of a pipe wall.

pressure field $P^{\prime}$ for the six flow rates considered here was computed and the results are shown in Fig. 9. These points were fit with a second-order polynomial and the fit equation is shown in the figure. $P^{\prime}$ is the standard deviation of the pipe wall pressure distribution and $Q$ is the flow rate in $\mathrm{m}^{3} / \mathrm{s}$. Since pressure is the primary source of energy transfer between the fluid and the structure, it is assumed that a similar relationship between flow rate and pipe acceleration will exist.

In the flow model, fully developed flow conditions were initiated and periodic boundary conditions were used to reduce the length of the pipe and the number of elements. The fully developed flow conditions were initiated using a mathematical model of isotropic turbulence developed by Goldin. ${ }^{16}$ In the structural solution however, the entire pipe was modeled such that the appropriate boundary conditions could be applied. Since the LES flow model was only a 10 $\mathrm{cm}$ domain, wall pressures extracted from the model were periodically copied 11 times to cover the structural domain. Due to computational and software constraints, 50 time steps were taken from the LES model to determine the structural response.

The final structural model consisted of a pipe of $L$ $=1.1 \mathrm{~m}, D=7.62 \mathrm{~cm}$, and a pipe wall thickness of $0.549 \mathrm{~cm}$. The $1.1 \mathrm{~m}$ length corresponds to supports on the test section. Approximately 11000 shell type elements were employed and the material type was set to AISI 304 steel. The boundary conditions for the structure were set as simply supported on one end with the other end restrained in the transverse direction but free along the axis of the pipe.

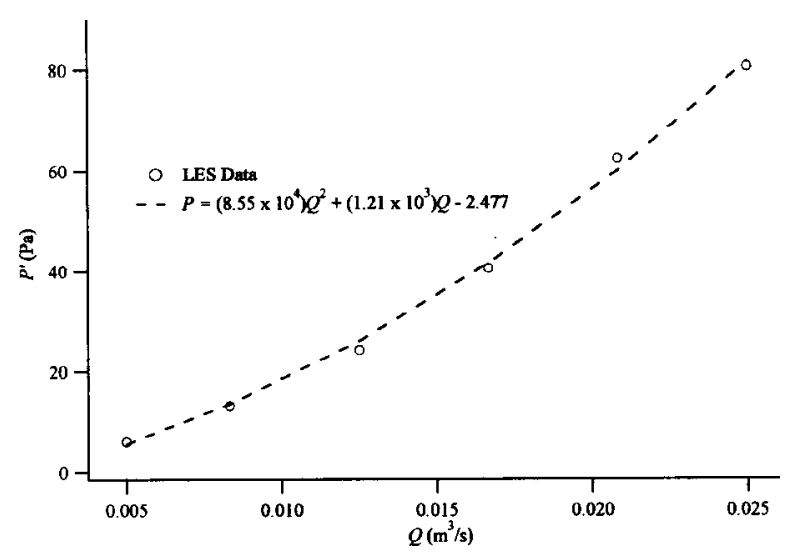

FIG. 9. Standard deviation of the pressure fluctuations on the pipe surface vs flow rate from LES model. 


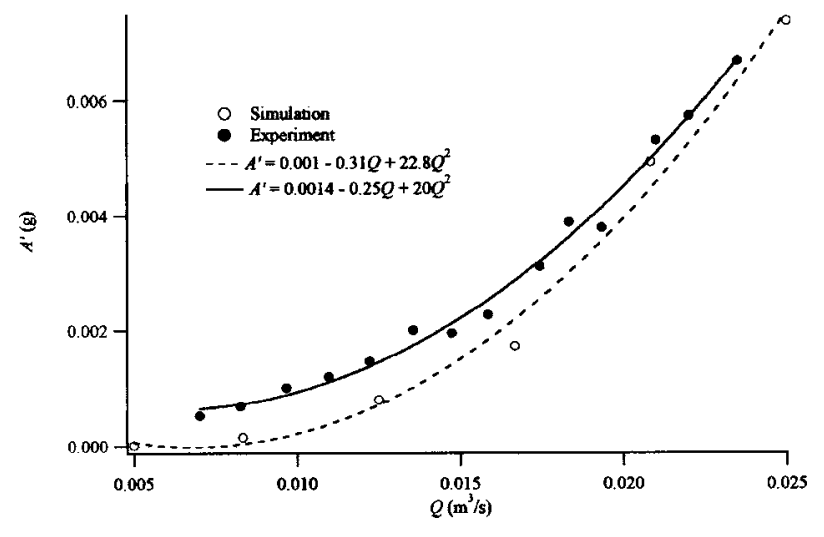

FIG. 10. $A^{\prime}$ as a function of $Q$ for flow through a steel pipe with an inside diameter of $7.62 \mathrm{~cm}$, from both experiment and an LES-structural model simulation.

After the deflections for the various flow rates were determined in the structural model, the acceleration of the pipe at a point was determined by computing the second temporal derivative of the pipe wall position. The standard deviation of all accelerations over the entire structure and time domain were then determined. This is plotted as a function of the volumetric flow rate in Fig. 10. It should be noted that these acceleration values are within the measurement range and resolution of many piezoelectric accelerometers. This plot is similar to the experimental work presented in Sec. II. Also shown in the figure are experimental data corresponding with corresponding pipe material, diameter, wall thickness, and length, in addition to least-squares curve fits to both sets of data. This plot shows that the data follows a similar trend for both the experimental and numerical simulations. The experimental data are initially at a higher level but both the simulation and experimental data merge at higher flow rates. By comparison with other experimental results, the contribution of the turbulent flow to the pipe vibration at low flow rates is a rather small component of the overall pipe vibration. However, as the flow rate is increased the turbulent flow induced vibration becomes a more significant component of the total response. Although great care was taken to match the experimental and numerical models in terms of modeling parameters such as boundary conditions and material properties, it is the opinion of the authors that the results of the two techniques are surprisingly similar and that absolute values should not be compared. However, the trends in both are clearly identified and accurately represented by the two models.

From this research, it was also concluded that the pressure fluctuations on the pipe wall have a near quadratic relationship with the flow rate. Furthermore, both the experimental results and the numerical modeling show that there is a definite relationship between the acceleration of the pipe (pipe vibration) and the flow rate. These last two concepts open possible avenues for the development of a nonintrusive flow sensor. This research has a direct impact on the geothermal, nuclear, and other fluid transport industries in the potential development of a nonintrusive flow sensor.

\section{ACKNOWLEDGMENT}

Funding for this research was provided by DOE through the Idaho National Engineering and Environmental Laboratory.

\section{NOMENCLATURE}

$A^{\prime} \quad$ Standard deviation of pipe vibration or acceleration

$A_{c} \quad$ Pipe cross-sectional area

$C_{s} \quad$ Smagorinsky constant

$D \quad$ Wall normal distance

$D \quad$ Pipe diameter

$f \quad$ Darcy friction factor

$g$ Gravitational constant

$L \quad$ Pipe length

$Q \quad$ Volume flow rate

$P \quad$ Pressure

$P_{f} \quad$ Pressure fluctuations from mean value

$P^{\prime} \quad$ Standard deviation of pipe wall pressure distribution

$\tilde{P} \quad$ Time averaged pressure

Re Reynolds number, $\operatorname{Re}=\rho_{w} V D / \mu$

$S_{i j} \quad$ Strain rate tensor

$t \quad$ Pipe wall thickness

$u \quad$ Streamwise velocity component

$u_{i} \quad$ Velocity vector

$\tilde{u}_{i} \quad$ Time averaged velocity vector

$u_{\tau} \quad$ Friction velocity, $u_{\tau}=\sqrt{\tau_{w} / \rho}$

$V \quad$ Volume of a computational cell

$V \quad$ Average pipe velocity

$x_{i} \quad$ Position vector

$y^{+} \quad$ Inner wall normal coordinate, $y^{+}=y \sqrt{\tau_{w} / \rho} / \nu$

$\varepsilon \quad$ Surface roughness

$\kappa \quad$ von Karman Constant

$\lambda \quad$ Subgrid mixing length

$\mu \quad$ Fluid viscosity

$\mu_{t} \quad$ Turbulent eddy viscosity

$\nu \quad$ Kinematic viscosity

$\rho \quad$ Fluid density

$\rho_{m} \quad$ Density of pipe material

$\rho_{w} \quad$ Density of water

$\tau_{i j} \quad$ Stress Tensor

$\tau_{w} \quad$ Wall shear stress

${ }^{1}$ R. P. Evans and L. D. Goodrich, 38th ISA International Instrumentation Symposium, Paper No. 92,0178 (Instrument Society of America, Las Vegas, Nevada, 1992).

${ }^{2}$ G. D. Lassahn, "LOFT Experimental Measurements Uncertainty Analysis-Methodology and Summary,” 1, NUREG/CR-0169, EGG-2037 (Idaho National Engineering and Environmental Laboratory, Idaho Falls, ID, 1983).

${ }^{3}$ R. Evans, J. Blotter, and A. Stephens, Determination of Flow Parameters in Two-Phase Flow Through Analysis of Flow-Induced Noise on an Accelerometer Signal; Geothermal Mass Flow Measurement Feasibility Report, Internal Report (Idaho National Engineering and Environmental Laboratory, Idaho Falls, ID, 1998).

${ }^{4}$ Robert P. Evans, 45th International Instrumentation Symposium, Albuquerque, $N M$ (ISA-International Society for Measurement and Controls, 1999), Vol. 1.

${ }^{5}$ N. Saito, H. Miyano, and S. Furukawa, Pressure Vessels and Piping Conference, Nashville, TN, 1990, Vol. 194, p. 233.

${ }^{6}$ C. Durant, G. Robert, P. Filippi, and P. Mattei, J. Sound Vib. 229, 1115 (2000).

${ }^{7}$ C. Durant and G. Robert, Turbulence and Combustion 61, 55 (1998). 
${ }^{8}$ B. J. Brevart and C. Fuller, J. Sound Vib. 167, 149 (1993).

${ }^{9}$ Young-Key Kim and Yang-Hann Kim, J. Acoust. Soc. Am. 100, 717 (1996).

${ }^{10}$ J. Eggels, Ph.D. thesis, Delft University, Delft, Netherlands, 1994.

${ }^{11}$ Stephen B. Pope, Turbulent Flows (Cambridge University, New York, 2000).

${ }^{12}$ M. Lesieur and O. Metais, Annu. Rev. Fluid Mech. 28, 45 (1996).
${ }^{13}$ FLUENT v5.5 Users Manual (Fluent Inc., Lebanon, NH, 2002), Chap. 9, pp. $40-60$.

${ }^{14}$ William Morrow Kays and Michael Crawford, Convective Heat and Mass Transfer, 3rd ed. (McGraw-Hill, New York, 1993), pp. 192-253.

${ }^{15}$ R. W. Fox, A. T. McDonald, and P. J. Pritchard, Introduction to Fluid Mechanics, 6th ed. (Wiley, New York, 2004).

${ }^{16} \mathrm{G}$. Goldin, FLUENT, June 2002. 